\title{
A New Method to Evaluate Acetabular Bone Defect in Patients With Crowe Type $₫$ and Type $\rrbracket$ Developmental Dysplasia of the Hip: Acetabular Reconstruction Based on Three-dimensional Reconstruction Images
}

\section{Xinggui Wen}

China-Japan Union Hospital of Jilin University

Jianlin Zuo

China-Japan Union Hospital of Jilin University

Tong Liu

China-Japan Union Hospital of Jilin University

Zhongli Gao

China-Japan Union Hospital of Jilin University

Jianlin Xiao ( $\square$ xiaojianlin10@jlu.edu.cn )

China-Japan Union Hospital of Jilin University

\section{Research Article}

Keywords: Developmental dysplasia of the hip, Acetabular reconstruction, High hip center technique, Bone defects distribution map

Posted Date: June 1st, 2021

DOI: https://doi.org/10.21203/rs.3.rs-557768/v1

License: (c) (1) This work is licensed under a Creative Commons Attribution 4.0 International License. Read Full License 


\section{Abstract}

This study evaluated the cup coverage(CC) of acetabular cup models with different sizes in patients with Crowe type $\otimes$ and type $\triangle \mathrm{DDH}$ by the high hip center technique $(\mathrm{HHC})$, the results shows that the $\mathrm{CC}$ of cup sizes with $38 \mathrm{~mm}, 40 \mathrm{~mm}, 42 \mathrm{~mm}, 44 \mathrm{~mm}, 46 \mathrm{~mm}, 48 \mathrm{~mm}$, and $50 \mathrm{~mm}$ increased by $21.24 \%, 21.58 \%$, $20.86 \%, 20.04 \%, 18.62 \%, 17.18 \%$, and $15.42 \%(P<0.001)$ respectively as the cups elevated from the true acetabula until the maximum coverage were achieved. What surprising is the mean $\mathrm{CC}$ at the true acetabula were $77.85 \%, 76.71 \%, 75.73 \%, 74.56 \%, 73.68 \%, 72.51 \%$, and $71.75 \%$, respectively.

So, we draw a distribution frequency map of uncovered portion of all 44-mm cups at the true acetabula to illustrate the distribution of bone defects of the acetabulum. The map shows that $95 \%$ of type $\nabla$ and type $\triangle D D H$ acetabula had bone defects in posterosuperior, and $60 \%$ were located outside the force line of the hip joint.

Here comes to a conclusion that acetabular cups can meet the $\mathrm{CC}$ of more than $70 \%$ at the true acetabulum, and $60 \%$ of Crowe type $\otimes$ and type $\triangle D D H$ patients can obtain satisfactory $C C$ at the true acetabulum by using a 44-mm cup without additional operation.

\section{Introduction}

The best approach for placement of the acetabular component in total hip arthroplasty (THA) remains controversial for patients with developmental dysplasia of the hip (DDH), especially for those with Crowe type $\nabla$ and type $\otimes$. When the acetabular cup is placed at the level of the true acetabulum, the bone defect caused by acetabular dysplasia may result in poor cup coverage (CC) in $\mathrm{THA}^{1,2}$, thus leading to unsatisfactory initial stability and low long-term survival rate ${ }^{3-5}$. In order to achieve adequate $\mathrm{CC}$, several studies $^{6-8}$ have recommended bone grafting, the medial protrusio technique, and the use of extra-small cups at the true acetabulum to during acetabular reconstruction. However, it remains disputable whether these techniques are helpful for reconstructing the acetabulum in DDH patients, because these techniques have various complications and uncertain long-term survival rates ${ }^{9-11}$.

The high hip center $(\mathrm{HHC})$ technique placing the acetabular cup above the true acetabulum during acetabular reconstruction, is suitable for DDH patients, because it can make full use of the bone above the true acetabulum to ensure adequate host bone coverage of the cup ${ }^{12,13}$. In addition, the HHC technique is also associated with some advantages such as reduced additional surgery and shortened operation time ${ }^{13}$. Based on three-dimensional (3D) reconstruction technology, several studies have shown that the $\mathrm{HHC}$ technique can greatly increase the bone coverage of the cup ${ }^{14-16}$, that may result in significant increases in the initial stability of the cup, bone ingrowth, and long-term survival. Our previous study on morphological features of the true acetabulum in DDH patients shows that the bone stock at the

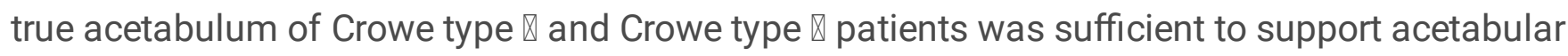
reconstruction, but for type $\otimes$ and type $\otimes$ patients, additional surgery may be required for in situ acetabular 
reconstruction ${ }^{17}$. However, these studies did not conduct in-depth study on acetabular reconstruction evaluation of type $₫$ and type $\otimes D D H$.

In 2009, Armitage et $\mathrm{al}^{18}$. analyzed the distribution of fracture line with 3D CT of scapula, and proposed the concept of fracture map. In the recent years, many studies reported the analysis of different fracture maps $^{19-21}$. To our knowledge, there is no study introduce the concept of fracture map into the field of joint replacement and related research, and investigate the distribution of acetabular bone defects in patients with type $\otimes$ and type $\otimes D D H$ from the perspective of uncovered acetabular cup.

Since a 44-mm cup is the smallest one available for ceramic-ceramic cementless prosthesis, some studies regard $44 \mathrm{~mm}$ cup as a standard cup ${ }^{15,16}$. In the present study, we firstly evaluated the CC of acetabular cup models with different sizes in patients with Crowe type $₫$ and type $\otimes D D H$ using the HHC technique to reconstruct the acetabulum, then analyzed the $\mathrm{CC}$ of host bone in all hips when reconstructing acetabulum at the true acetabulum with 44-mm acetabular cup models. Finally, evaluated the uncoverage of cup models with 44-mm diameter at the true acetabulum, and draw a 3D distribution map of the uncovered portions of the acetabular cup that can represent the bone defect of the true acetabulum.

\section{Materials And Methods}

\section{Patients}

This study was approved by the Institutional Review Board, Ethics Committee of China-Japan Union Hospital (IRB No: 2016ks001). All patients had signed informed consent before X-ray and computer tomographic (CT) examination. All operations and experiments were carried out in strict accordance with the relevant regulations and guidelines. The present study included 49 hips of 39 patients ( 3 males and 36 females) who underwent hip X-ray and CT examination in our hospital. According to Crowe classification ${ }^{1}, 28$ hips were classified as type $\Downarrow$, and 21 hips were type $\otimes$. All patients did not have hip diseases including avascular necrosis of femoral head and infection or history of hip surgery, such as pelvic osteotomy, bone grafting, and osteotomy of the femur. The demographic data are shown in Table 1. 
Table 1

Demographic characteristics

\begin{tabular}{|c|c|}
\hline Characteristic & Value \\
\hline Number of patients (hips) & $39(49)$ \\
\hline Gender (male/female) & $3 / 36$ \\
\hline Age $(\text { Years })^{\rrbracket}$ & $48.92 \pm 10.69$ \\
\hline Height $(\mathrm{cm})^{\rrbracket}$ & $158.59 \pm 5.78$ \\
\hline Weight (kg) ${ }^{\llbracket}$ & $60.74 \pm 9.65$ \\
\hline BMI (Body Mass Index, $\left.\mathrm{kg} / \mathrm{m}^{2}\right)^{\llbracket}$ & $24.11 \pm 3.38$ \\
\hline Unilateral(left/right) & $29(12 / 17)$ \\
\hline Bilateral & 10 \\
\hline \multicolumn{2}{|l|}{ Crowe type (number of hips) } \\
\hline ૫ & 28 \\
\hline$\square$ & 21 \\
\hline${ }^{\circledR}$ Values are expressed as means & SD. \\
\hline
\end{tabular}

CT scan and 3D reconstruction

CT scans of the entire pelvis were performed by Toshiba Aquilion CT scanner $(120 \mathrm{kVp}, 320 \mathrm{~mA}, 512 \times 512$ matrix, and 0.5-mm slice thickness). All CT data were saved in Digital Imaging and Communications in Medicine (DICOM) format, then imported into Mimics 17.0 software (Materialise) and used to reconstruct $3 \mathrm{D}$ images for each pelvis. The position of 3D reconstruction images were adjusted according to the anterior plane of the pelvis determined by the anterior superior iliac spine and pubic tubercle ${ }^{17}$.

Simulating Implantation of the Acetabular Cup Model

We used a set of acetabular cup models created in our previous study ${ }^{17}$. The diameter of the cups ranged from 38-50 mm in 2-mm increments. All cups had a shell thickness of $0.1 \mathrm{~mm}$. These models were imported into the Mimics software in the STL (stereolithography) format, and the total surface area $\left(S_{t}\right)$ was available. The cups were implanted into the reconstructed 3D images of the pelvis using the Mimics software, and the method of simulating acetabular cup implantation in THA was mentioned in our previous study ${ }^{17}$. The position of all the cups was set at $40^{\circ}$ abduction and $15^{\circ}$ anteversion constantly (Figure. 1A).

Measurement of CC 
To measure CC, the cups were placed at the true acetabulum firstly ${ }^{22}$, and then elevated upward in 2-mm increments until it reached $50 \mathrm{~mm}$ (Figure. 1B). At each elevation, the cup models were slightly adjusted on coronal and transverse sections to achieve the max CC. After the cup models were placed in the 3D reconstruction image of the pelvis, the surface area of uncovered portion $\left(\mathrm{S}_{\mathrm{u}}\right)$, can read by the Boolean operation function of Mimics. The CC of cups with different diameters (range from $38 \mathrm{~mm}$ to $50 \mathrm{~mm}$ ) at each elevation was calculated by the following formula:

\section{$\mathrm{CC}=\left(S_{t}-S_{u}\right) / S_{t} \times 100 \%$}

Mapping the Uncovered Portions of Acetabular Cups

The uncovered portions of all 44-mm cups at the true acetabula were imported into Magics 22.03 software (Materialise) in STL format to overlap onto a complete cup model. Uncovered portion of all 44$\mathrm{mm}$ cups were superimposed to create a compilation of uncoverage on a complete cup model serving as a representation of the exposed portion of acetabular cups in THA. The overlap of all uncoverage of cups were suited to build a distribution map of frequency based on the density of uncovered portion of cups, and then calculated the distribution frequency.

Analysis of defect distribution

Making a vertical line through the rotation center of the cup, and then built a plane perpendicular to the anterior plane of pelvis based on this line. So that, the distribution frequency of the uncovered portion of the cups outside the gravity line of hip joint can be calculated. And we can calculate the probability of taking additional surgery such as bone graft in acetabular reconstruction at the true acetabulum in patients with Crowe type $₫$ and type $\otimes D D H$.

Statistical Analysis

All data are expressed as means \pm standard deviation (SD). The paired $t$ test was used to evaluate the differences between the $\mathrm{CC}$ at the true acetabulum and the maximum $\mathrm{CC}$. Pearson's correlation coefficient was used to evaluate the correlation between CC and the elevated height of the cup. All statistical analyses were performed using SPSS 21.0 software (SPSS, Chicago, IL, USA). Statistical significance was defined as $\mathrm{P}<0.05$.

\section{Results}

The CC of all cups with different sizes at the true acetabulum were greater than $70 \%$, and as the acetabular cups elevated from the true acetabulum, the $\mathrm{CC}$ gradually increased until reaching the maximum, then decreased gradually even below $70 \%$ (Fig. 2). When CC reaches the maximum and decreases to less than $70 \%$, the corresponding elevated height of cups decreases with the increase of cup size. 
CC of all cups at the true acetabulum are greater than $71 \%$, all the cups reached the maximum CC greater than $87 \%$ after elevating from the true acetabulum, and the maximum CC was significantly different from that at the true acetabulum (Table 2). When CC reached the mean maximum value of $93.96 \%$, the cup was elevated up to a mean height of $23.76 \mathrm{~mm}$ from the true acetabulum and the CC increased by $19.28 \%(P<0.001)$. The mean CC of $44-\mathrm{mm}$ cups at the true acetabulum is $74.56 \%$, and it reaches the maximum of $94.61 \%$ after elevating $24.16 \mathrm{~mm}$ from the true acetabulum.

Table 2

The $\mathrm{CC}$ at the true acetabulum and the maximum CC

\begin{tabular}{|c|c|c|c|c|}
\hline $\begin{array}{l}\text { Cup size } \\
\text { (mm) }\end{array}$ & $\begin{array}{l}\text { CC at true acetabulum } \\
(\%)^{囚}\end{array}$ & $\begin{array}{l}\text { Maximum CC } \\
(\%)^{囚}\end{array}$ & $\begin{array}{l}\text { Difference } \\
(\%)^{\bigotimes}\end{array}$ & $\begin{array}{l}\text { Elevated height } \\
(\mathrm{mm})^{\rrbracket}\end{array}$ \\
\hline 38 & $77.85 \pm 7.88$ & $99.09 \pm 2.21$ & $21.24 \pm 8.27^{\otimes}$ & $29.14 \pm 5.85$ \\
\hline 40 & $76.71 \pm 7.28$ & $98.30 \pm 3.07$ & $21.58 \pm 7.62^{\llbracket}$ & $27.84 \pm 5.51$ \\
\hline 42 & $75.73 \pm 6.40$ & $96.59 \pm 4.43$ & $20.86 \pm 6.94 \rrbracket$ & $25.96 \pm 5.77$ \\
\hline 44 & $74.56 \pm 6.21$ & $94.61 \pm 5.67$ & $20.04 \pm 6.88^{\rrbracket}$ & $24.16 \pm 6.16$ \\
\hline 46 & $73.68 \pm 6.27$ & $92.30 \pm 7.17$ & $18.62 \pm 6.54^{\bigotimes}$ & $21.76 \pm 5.83$ \\
\hline 48 & $72.51 \pm 6.65$ & $89.69 \pm 8.51$ & $17.18 \pm 7.01^{\llbracket}$ & $19.59 \pm 6.51$ \\
\hline 50 & $71.75 \pm 7.23$ & $87.17 \pm 9.55$ & $15.42 \pm 7.07^{\square}$ & $17.88 \pm 7.12$ \\
\hline Mean & $74.69 \pm 7.11$ & $93.96 \pm 7.52$ & $19.28 \pm 7.46^{\rrbracket}$ & $23.76 \pm 7.22$ \\
\hline \multicolumn{5}{|c|}{${ }^{\circledR}$ Values are presented as mean \pm SD. } \\
\hline${ }^{\nabla} \mathrm{P}<0.0$ & ed t test & & & \\
\hline
\end{tabular}

Figure 3 shows the relationship between the $\mathrm{CC}$ value and the elevated height when the cup was elevated from the true acetabulum until the mean maximum value of $\mathrm{CC}$ was reached. The $\mathrm{CC}$ values were positively correlated with elevated height for cups with the sizes of $38,40,42,44,46,48$, and $50 \mathrm{~mm}$, the Pearson Correlation Coefficient were 0.9922, 0.9933, 0.9918, 0.9972, 0.9879, 0.9717, and 0.9645 respectively, indicating that the correlation between $\mathrm{CC}$ and elevated height was almost linear in all cups. The mean elevated height that $\mathrm{CC}$ reach the maximum decrease with the increase of cup size.

The exposed portion of cups mainly ( $>60 \%$ ) concentrated in the posterosuperior, and a small area located in the area between 11 and 12 o'clock on almost all the cups are not covered by the host bone, as shown in crimson (Fig. 4). There is $50 \sim 70 \%$ uncovered portion concentrated in the anteroinferior of cup, and surprisingly, about 10\% 25\% uncovered portion was in the medial of cup. Uncovered portions of several special cases make the frequency of $5 \%$ 10\% concentrated in the posteromedial of cup. 
As shown in Fig. 4, over $60 \%$ uncovered portion of cup was outside the gravity line of hip, so it can be inferred that when the 44-mm cup is used to reconstruct acetabulum at the true acetabulum for Crowe type $\varangle$ and type $\varangle \mathrm{DDH}$ patients, there is about $40 \%$ uncovered portion of cup locate within the gravity line of hip, and these hips may require additional surgery to ensure the initial CC in THA.

\section{Discussion}

According to previous studies ${ }^{1}$, for Crowe type $₫$ and type $₫ D D H$ patients, it is difficult to achieve satisfactory CC when reconstructing the acetabulum due to the shallow acetabulum and bone defects. Placing the cup in the bone stock above the true acetabulum with sufficient bone mass can increase the $C C$ and ensure the initial stability of the acetabular cup ${ }^{13-16,23}$. Here, we found that elevating the cups with different sizes to $23.76 \mathrm{~mm}$ above the true acetabulum increased the CC by $19.28 \%$ during acetabular reconstruction for patients with Crowe type $\triangle$ and type $\triangle D D H$, which is consistent with the findings of previous studies ${ }^{12,14-16,23}$. In addition, the mean elevated height identified in this study meets the safety range determined by Hirakawa et al. ${ }^{24}$, who recommended that the cup should be placed $<35$ $\mathrm{mm}$ vertically from the interteardrop line.

Sufficient host bone coverage of the acetabular cup is required to achieve satisfactory long-term implant survival for DDH patients following THA. Many studies have demonstrated that HHC technique is a feasible method for reconstructing the acetabulum at the level of true acetabulum in DDH patients ${ }^{14-16}$. In this study, except for the 48-mm and 50-mm cups, other cups achieved the mean CC of greater than $90 \%$ after elevating from true acetabulum. Many studies have shown that $\mathrm{HHC}$ technique does not cause aseptic loosening of cups, and the outcomes after medium and long-term follow-ups are satisfactory ${ }^{23}$, ${ }^{25-28}$. Therefore, we believe that the HHC technique is a valid method to reconstruct acetabulum for DDH patients.

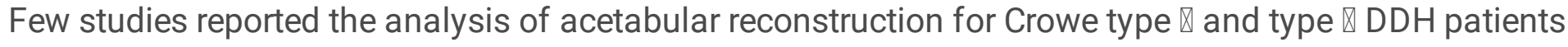
based on CT of pelvis and acetabular cup prosthesis model. Komiyama ${ }^{29}$ used a cup with diameter of $50 \mathrm{~mm}$, and positioned the cup with an inclination of $40^{\circ}$ and an anteversion of $20^{\circ}$ to simulate acetabular reconstruction in 32 patients with Crowe type $₫$ and type $\otimes D D H$, the cup center-edge (Cup-CE) angle was used to evaluate CC. The result shows that, at the anatomical hip center, the mean Cup-CE was $-4.3 \pm 11.8^{\circ}, 13$ hips (40.6\%) satisfied Cup-CE $\geq 0^{\circ}$, and the Cup CE angle $\geq 0^{\circ}$ was used as the cut off value for the required bone coverage in their study, which was approximately equal to bone coverage of acetabular cup $\geq 60 \%$. Xu et al ${ }^{16}$. reported acetabular reconstruction for Crowe IV DDH patients with 44$\mathrm{mm}$ cup at a position of $40^{\circ}$ abduction and $20^{\circ}$ anteversion, the simulated mean in situ 3D CC was

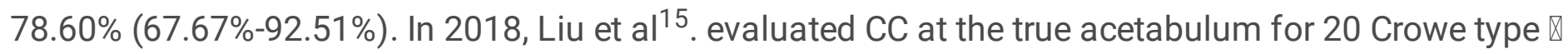
DDH hips with $44 \mathrm{~mm}$ cup model, the inclination was set to $45^{\circ}$ constantly, and three anteversion groups with $0^{\circ}, 5^{\circ}$, and $10^{\circ}$ were set. The CC was $65.87 \% \pm 7.82 \%, 67.77 \% \pm 8.02 \%$, and $68.98 \% \pm 6.97 \%$ respectively. To our knowledge, the present stud is the first one to evaluate $\mathrm{CC}$ at the true acetabulum for Crowe type $\varangle$ and $\triangle D D H$ patients based on $3 \mathrm{D}$ reconstruction images and the results are different from 
previous views and studies, that is, using cups with diameter of $38 \mathrm{~mm}, 40 \mathrm{~mm}, 42 \mathrm{~mm}, 44 \mathrm{~mm}, 46 \mathrm{~mm}$, $48 \mathrm{~mm}$, and $50 \mathrm{~mm}$ to reconstruct acetabulum, the cups can obtain a mean 3D bone coverage of $74.69 \%$ at the true acetabulum. According to the existing literatures ${ }^{30-32}$, no additional operation is required to ensure the initial stability of cups during acetabular reconstruction if CC is greater than $70 \%$.

This study analyzed CC of $44 \mathrm{~mm}$ cups at the true acetabulum and the distribution of uncovered portion in this study. Many existing studies ${ }^{2}, 14,15,17,33,34$ have described the bone defect of the true acetabulum in DDH patients from the perspective of pelvic morphology, but few studies analyzed the uncoverage from the perspective of acetabular cup. We all know that setting a proper position of the acetabular cup is the only thing we can do during the THA, so it is the most important to carry out relevant research from the perspective of the acetabular cup. The present study is the first one that analyzed the uncovered portion of cups in THA and drew a map of uncovered portion of cups that represents bone defects of true acetabulum. From this map, we can intuitively see the distribution and probability of the uncovered portion of the acetabular cup. The results show that $40 \%$ of the uncovered portion of cups are located inside the gravity line of hip, we believe that if the uncovered portion of the cup is located inside the gravity line of the hip joint, hearing forces on the acetabular cup may lead to early loosening ${ }^{4}$. So, we can predict that about $60 \%$ of type $\otimes$ and type $\triangle \mathrm{DDH}$ patients can obtain satisfactory $\mathrm{CC}$ without additional operation when reconstructing acetabula at the true acetabulum with 44-mm cups. This proportion is not very large, but it is really a surprising finding. That is, during acetabular reconstruction for type $\otimes$ and type $\triangle D D H$ patients, if careful preoperative design is carried out, in many patients, placing acetabular cup at the true acetabulum can also obtain good CC and initial stability can be ensured.

On the other hand, from the results of this study, if the acetabular bone defect is too large and bone grafting is necessary, the bone defect distribution map can be used as a guide for orthopedic surgeons to determine the location of bone grafts during preoperative design.

\section{Conclusion}

Based on the 3D reconstruction technology and surgical simulation technology, although the HHC

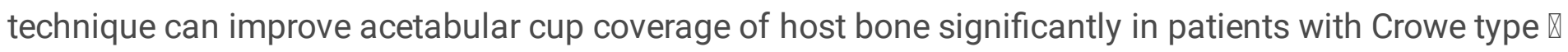
and type $\triangle D D H$, all cups can obtain a satisfactory CC (more than $70 \%$ ) at the true acetabula during acetabular reconstruction. A distribution map of the uncovered portions of the acetabular cup that can represent the bone defect of the true acetabulum was drawn, and from the map, about $60 \%$ of Crowe type $\triangle$ and type $\triangle \mathrm{DDH}$ patients can obtain satisfactory $\mathrm{CC}$ of host bone at the true acetabula by using a 44$\mathrm{mm}$ cup without additional operation.

\section{Declarations}

\section{Authors Contributions:}


$X G$ W, JL Z and JL X conceived the article. XG W and JL Z wrote the manuscript and the figures. XG W, JL $Z$, and $T L$ collected the data and designed the tables. JL Z, ZL G, and JL X performed literature search and provided valuable comments. All authors discussed the results and revised the manuscript. All authors contributed to the article and approved the final manuscript.

\section{Conflict of interest statement}

The authors declare no competing interests.

\section{Funding Statement.}

This work was supported by the funding from the National Nature Science Foundation of Youth in China (No: 81601907) 『China Postdoctoral Science Foundation (No: 2020M670863) \ Natural Science Foundation of Jilin Province (No: 20200201345JC).

\section{References}

1. Crowe J.F., Mani V.J., Ranawat C.S. Total hip replacement in congenital dislocation and dysplasia of the hip. J Bone Joint Surg Am.61(1):15-23. (1979)

2. Hartofilakidis G., Stamos K., Karachalios T., Ioannidis T.T., Zacharakis N., Athens G. Congenital hip disease in adults : classification of acetabular deficiencies and operative treatment with acetabuloplasty combined with total hip arthroplasty. J Bone Joint Surg Am.78(5):683-92. (1996)

3. Chougle A., Hemmady M.V., Hodgkinson J.P. Severity of hip dysplasia and loosening of the socket in cemented total hip replacement. J Bone Joint Surg Br.87(1):16-20. (2005)

4. Hartofilakidis G., Karachalios T. Total Hip Arthroplasty for Congenital Hip Disease. J Bone Joint Surg Am.86(2):242-50. (2004)

5. Hartofilakidis G., Stamos K., Karachalios T. Treatment of high dislocation of the hip in adults with total hip arthroplasty. Operative technique and long-term clinical results. J Bone Joint Surg Am.80(4):510-7. (1998)

6. Kim M., Kadowaki T. High Long-term Survival of Bulk Femoral Head Autograft for Acetabular Reconstruction in Cementless THA for Developmental Hip Dysplasia. Clin Orthop Relat Res.468(6):1611-20. (2010)

7. Dorr L.D., Tawakkol S., Moorthy M., Long W., Wan Z. Medial protrusio technique for placement of a porous-coated, hemispherical acetabular component without cement in a total hip arthroplasty in patients who have acetabular dysplasia. J Bone Joint Surg Am.81(1):83-92. (1999)

8. Paavilainen T., Hoikka V., Solonen K.A. Cementless total replacement for severely dysplastic or dislocated hips. J Bone Joint Surg Br.72(2):205-11. (1990)

9. Lee B.P., Cabanela M.E., Wallrichs S.L., Ilstrup D.M. Bone-graft augmentation for acetabular deficiencies in total hip arthroplasty - Results of long-term follow-up evaluation. $J$ Arthroplasty.12(5):503-10. (1997) 
10. Petsatodis G.E., Papadopoulos P.P., Papavasiliou K.A., Hatzokos I.G., Christodoulou A.G. Primary Cementless Total Hip Arthroplasty with an Alumina Ceramic-on-Ceramic Bearing. J Bone Joint Surg Am.92(3):639-44. (2010)

11. Shetty A.A., Sharma P., Singh S., Tindall A., Kumar S.V., Rand C. Bulk femoral-head autografting in uncemented total hip arthroplasty for acetabular dysplasia: Results at 8 to 11 years follow-up. $J$ Arthroplasty.19(6):706-13. (2004)

12. Hendricks K.J., Harris W.H. High Placement of Noncemented Acetabular Components in Revision Total Hip Arthroplasty: A Concise Follow-Up, at a Minimum of Fifteen Years, of a Previous Report. $J$ Bone Joint Surg Am.88(10):2231-6. (2006)

13. Bozic K.J., Freiberg A.A., Harris W.H. The High Hip Center. Clin Orthop Relat Res(420):101-5. (2004)

14. Gao Z.L., Xiao J.L., Zuo J.L., Liu P., Liu T. Cross-sectional Anatomy of Ilium for Guiding Acetabular Component Placement Using High Hip Center Technique in Asian Population. Chin Med J (Engl).128(12):1579-83. (2015)

15. Liu B., Gao Y.-H., Ding L., Li S.-Q., Liu J.-G., Qi X. Computed Tomographic Evaluation of Bone Stock in Patients with Crowe Type III Developmental Dysplasia of the Hip: Implications for Guiding Acetabular Component Placement Using the High Hip Center Technique. J Arthroplasty.33(3):915-8. (2018)

16. Xu J., Xu C., Mao Y., Zhang J., Li H., Zhu Z. Posterosuperior Placement of a Standard-Sized Cup at the True Acetabulum in Acetabular Reconstruction of Developmental Dysplasia of the Hip With High Dislocation. J Arthroplasty.31(6):1233-9. (2016)

17. Yang Y., Zuo J., Liu T., Xiao J., Liu S., Gao Z. Morphological Analysis of True Acetabulum in Hip Dysplasia (Crowe Classes I-IV) Via 3-D Implantation Simulation. J Bone Joint Surg Am.99(17):e92. (2017)

18. Armitage BM W.C., Tarkin IS, Schroder LK, Marek DJ, Zlowodzki M, Cole PA. Mapping of Scapular Fractures with Three-Dimensional Computed Tomography. J Bone Joint Surg Am.91(9):2222-8. (2009)

19. Zhan Y., Xie X., Dong M., He Q., Luo C. Two and Three-Dimensional CT Mapping of Hoffa Fractures. J Bone Joint Surg Am.99(21):1866-74. (2017)

20. Yun Y., Min Y., Chang Z., Zhao-Kui Y., Xin-An Y., Yue F. Mapping of 238 quadrilateral plate fractures with three-dimensional computed tomography. Injury.49(7):1307-12. (2018)

21. Turow A., Bulstra A.E., Oldhoff M., Hayat B., Bain G.I. 3D mapping of scaphoid fractures and comminution. Skeletal Radiol.49(10):1633-47. (2020)

22. Sariali E., Boukhelifa N., CATONNE Y., Moussellard H.P. Comparison of Three-Dimensional PlanningAssisted and Conventional Acetabular Cup Positioning in Total Hip Arthroplasty: A Randomized Controlled Trial. J Bone Joint Surg Am.98(2):108-16. (2016)

23. Murayama T., Ohnishi H., Okabe S., Tsurukami H., Mori T., Nakura N., et al. 15-year Comparison of Cementless Total Hip Arthroplasty With Anatomical or High Cup Placement for Crowe I to III Hip Dysplasia. Orthopedics.35(3):e313-8. (2012) 
24. Hirakawa K., Mitsugi N., Koshino T., Saito T., Hirasawa Y., Kubo T. Effect of Acetabular Cup Position and Orientation in Cemented Total Hip Arthroplasty. Clin Orthop Relat Res(388):135-42. (2001)

25. Kaneuji A., Sugimori T., Ichiseki T., Yamada K., Fukui K., Matsumoto T. Minimum Ten-Year Results of a Porous Acetabular Component for Crowe I to III Hip Dysplasia Using an Elevated Hip Center. $J$ Arthroplasty.24(2):187-94. (2009)

26. Nawabi D.H., MD... M.M. Durable Fixation Achieved With Medialized, High Hip Center Cementless THAs for Crowe II and III Dysplasia. Clin Orthop Relat Res.472(2):630-6. (2014)

27. Karaismailoglu B., Kaynak G., Can A., Ozsahin M., Erdogan F. Bilateral High Hip Center Provides Gait Parameters Similar to Anatomical Reconstruction: A Gait Analysis Study in Hip Replacement Patients With Bilateral Developmental Dysplasia. J Arthroplasty.34(12):3099-105. (2019)

28. Montalti M., Castagnini F., Giardina F., Tassinari E., Biondi F., Toni A. Cementless Total Hip Arthroplasty in Crowe III and IV Dysplasia: High Hip Center and Modular Necks. J Arthroplasty.33(6):1813-9. (2018)

29. Komiyama K., Nakashima Y., Hirata M., Hara D., Kohno Y., Iwamoto Y. Does High Hip Center Decrease Range of Motion in Total Hip Arthroplasty? A Computer Simulation Study. $J$ Arthroplasty.31(10):2342-7. (2016)

30. Rogers B.A., Garbedian S., Kuchinad R.A., Backstein D., Safir O., Gross A.E. Total Hip Arthroplasty for Adult Hip Dysplasia. J Bone Joint Surg Am.94(19):1809-21. (2012)

31. Jasty M., Anderson M.J., Harris W.H. Total hip replacement for developmental dysplasia of the hip. Clin Orthop Relat Res.311(311):40-5. (1995)

32. Schüller HM, Dalstra M, Huiskes R, RK M. Total hip reconstruction in acetabular dysplasia. A finite element study. J Bone Joint Surg Br.75(3):468-74. (1993)

33. Fujii M., Nakashima Y., Sato T., Akiyama M., Iwamoto Y. Acetabular Tilt Correlates with Acetabular Version and Coverage in Hip Dysplasia. Clin Orthop Relat Res.470(10):2827-35. (2012)

34. Liu R.Y., Wang K.Z., Wang C.S., Dang X.Q., Tong Z.Q. Evaluation of Medial Acetabular Wall Bone Stock in Patients with Developmental Dysplasia of the Hip Using a Helical Computed Tomography Multiplanar Reconstruction Technique. Acta Radiologica.50(7):791-7. (2009)

\section{Figures}



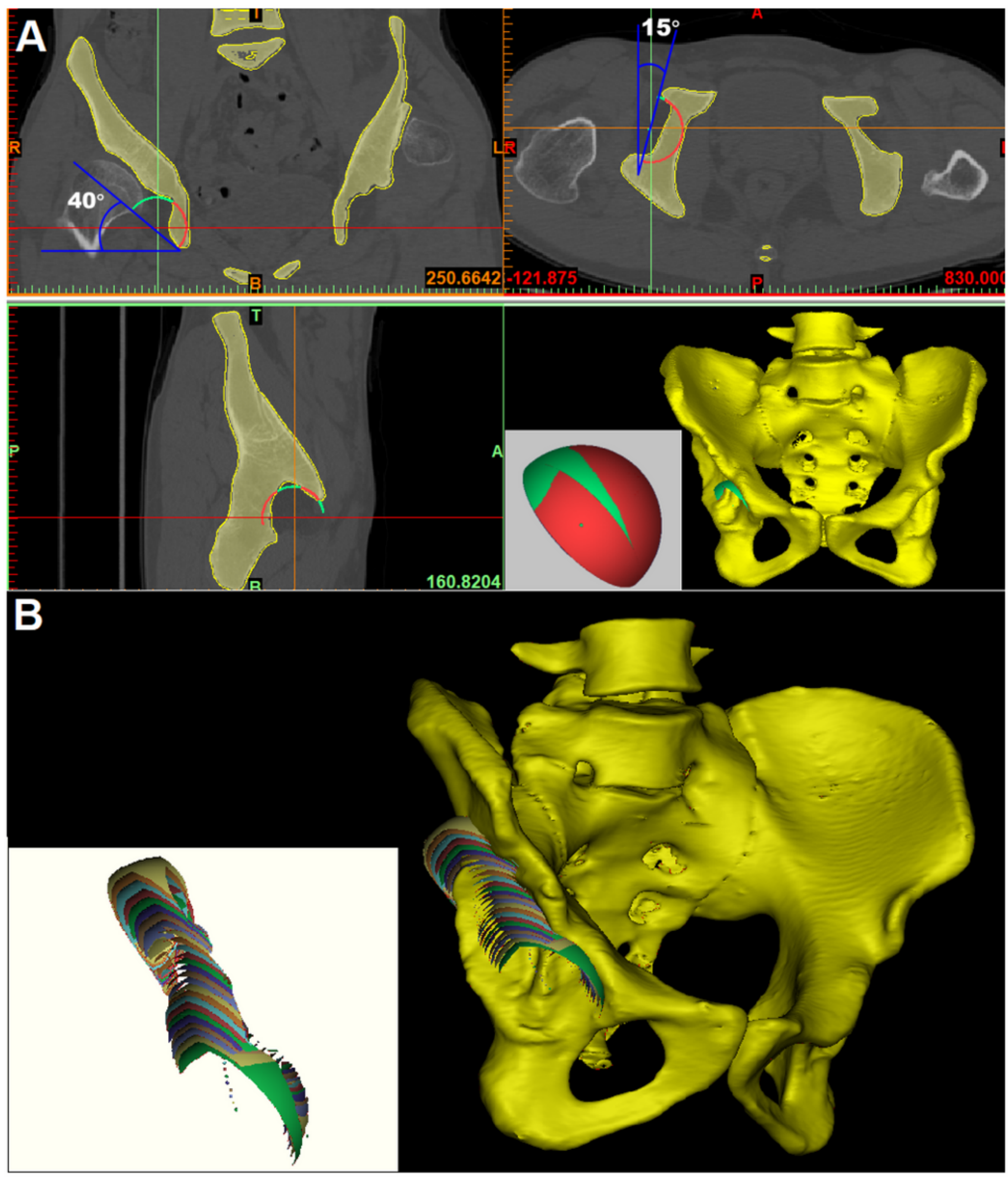

\section{Figure 1}

3D images of the pelvis reconstructed by Mimics software using complete CT data of the pelvis. A: The cup models were placed in the 3D reconstruction image of the pelvis to simulate acetabulum reconstruction in THA. The position of the cup was adjusted to $40^{\circ}$ of abduction and $15^{\circ}$ of anteversion on the coronal and transverse sections. The total surface area of the cup (St) can be read directly from the Mimics software. Boolean operation was used to obtain the uncovered area of cup (Su), which is 
indicated in green and covered area is in red. B: The uncovered portions of the cup with a diameter of $50 \mathrm{~mm}$ at different elevations are indicated in different colors.
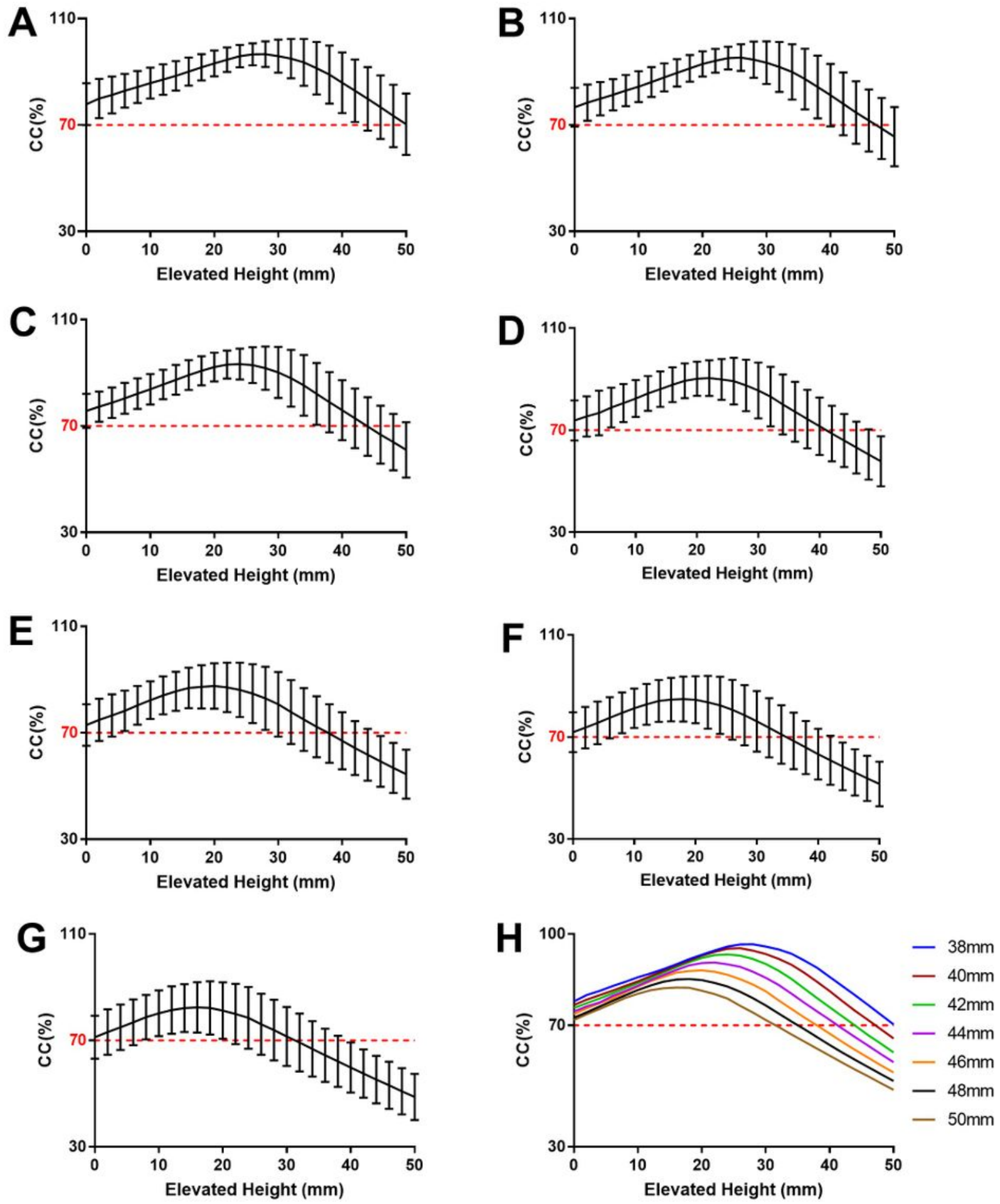

Figure 2

Changes in $\mathrm{CC}$ and the elevated height of the cup from the true acetabulum when for cup sizes of $38 \mathrm{~mm}$ (A), $40 \mathrm{~mm}(B), 42 \mathrm{~mm}(C), 44 \mathrm{~mm}(\mathrm{D}), 46 \mathrm{~mm}(\mathrm{E}), 48 \mathrm{~mm}(\mathrm{~F})$, and $50 \mathrm{~mm}(\mathrm{G})$. H. shows the relationship 
between the $\mathrm{CC}$ and the elevated height of cups with different sizes. With the increase of cup size, the elevated height of cup decreases gradually when the maximum CC was reached.

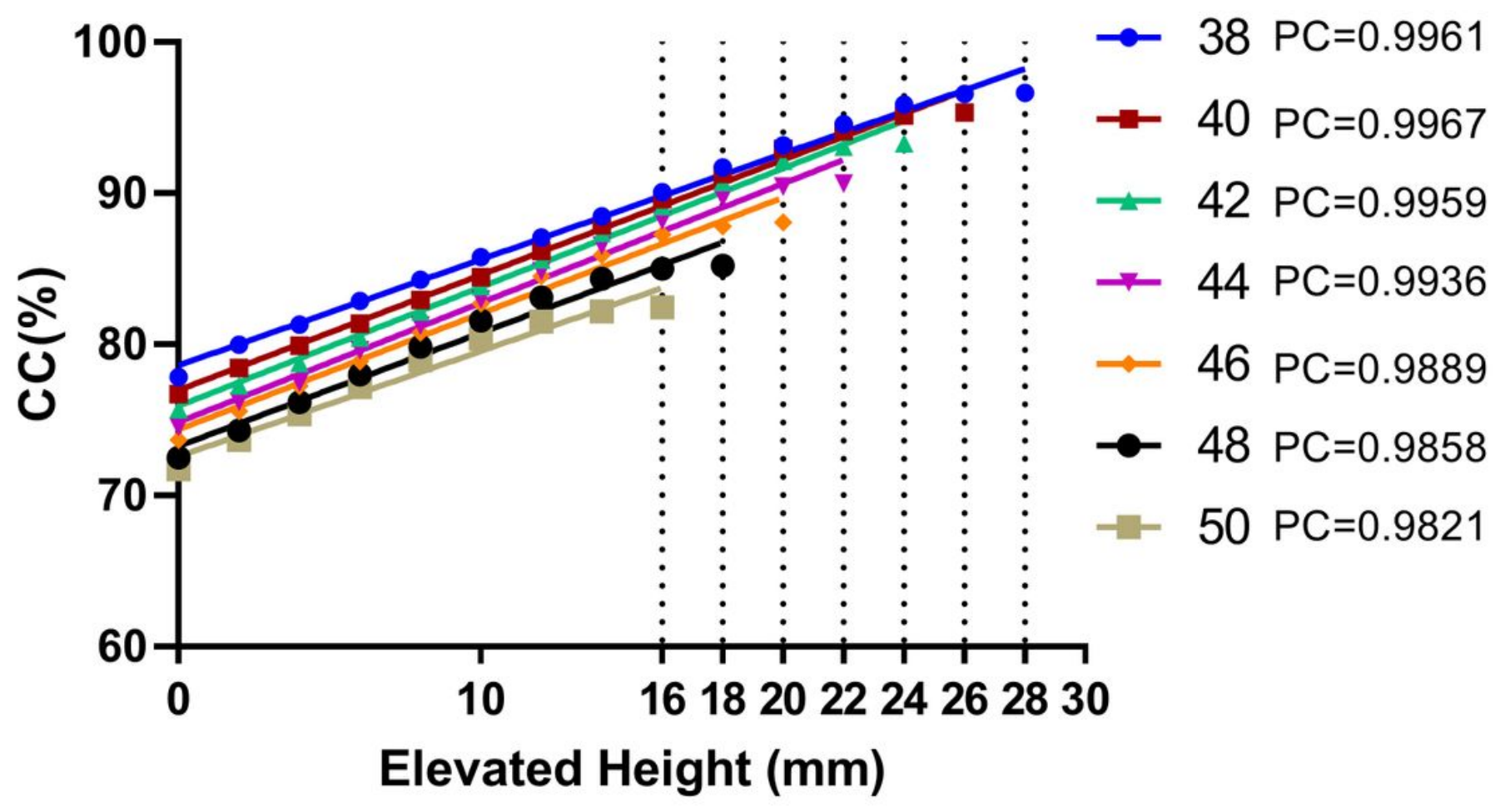

Figure 3

Relationship of the $\mathrm{CC}$ and elevated height of cups from the true acetabulum till the $\mathrm{CC}$ reached the maximum value. PC: Pearson Correlation Coefficient. 


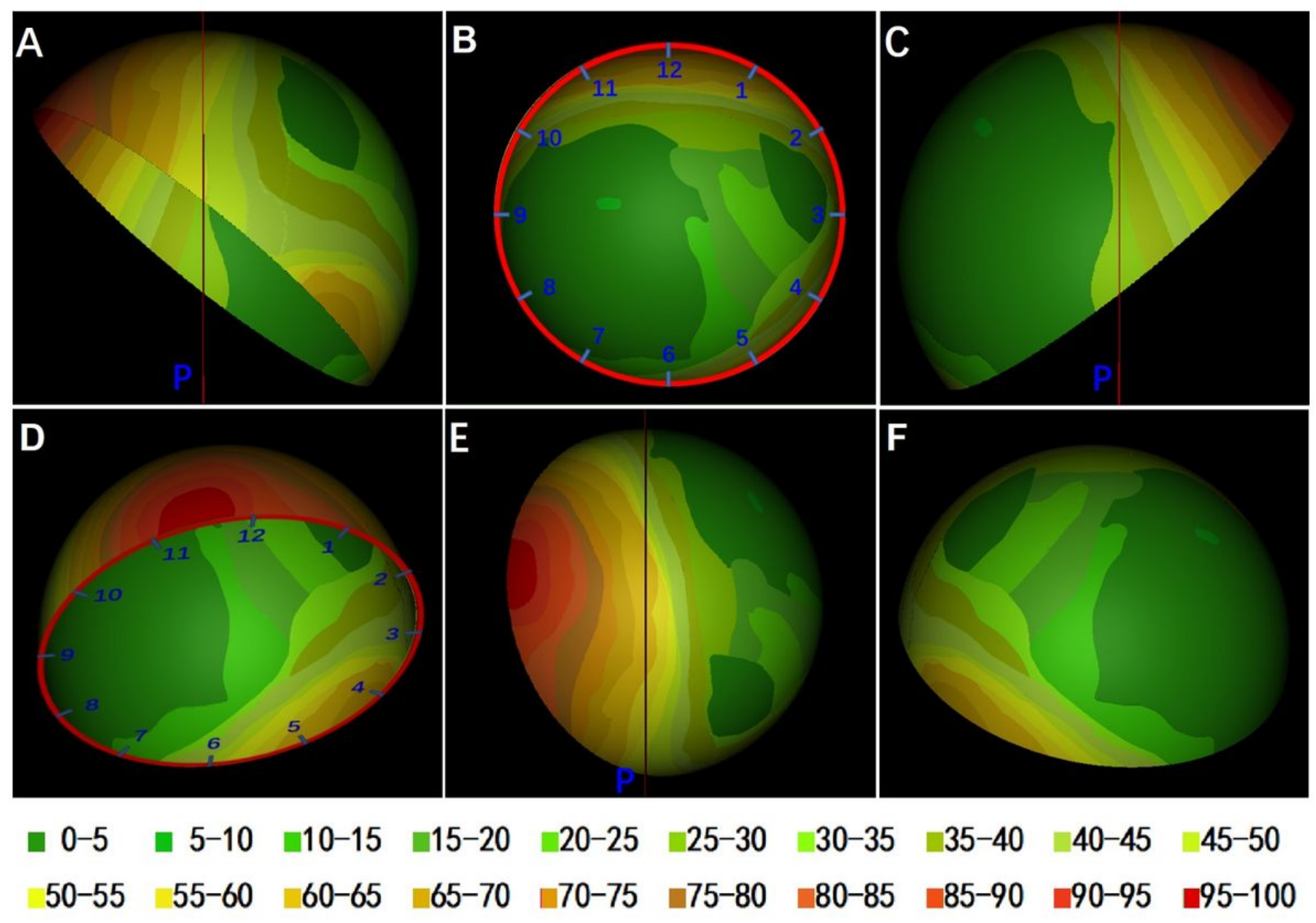

Figure 4

The distribution frequency of uncovered portion on intact cup is shown in different colors. The result is expressed as percentage with total of 49. A clock pattern was drawn on the cup to show the location of the uncovered portion. Builing a plane perpendicular to the anterior plane of pelvis through the rotation center of the cup. (A) Front view of the pelvis, the red line is a vertical line through the rotation center of the mortar cup. (B) Front view of the cup. (C) Rear view of the cup. (D) Vertical view of the cup. p: Vertical plane through the rotation center of the cup. 